\title{
NOTES ON A THEOREM ON CONVOLUTIONS
}

\author{
J. N. WHITELEY
}

1. Introduction. In these notes we prove first a refinement of Theorem 2 in [1], which will be our Theorem 1 . As an application of this result, in our Theorem 2, we obtain a series of inequalities for the coefficients of a real polynomial whose zeros lie relatively close together. This leads to Theorem 3 , in which, as a limiting case of Theorem 2, we obtain a result about real polynomials with zeros in a sector of angle $\pi / 2$. This last result could also be deduced from the GaussLucas Theorem on the zeros of the derivative of a polynomial, using reasoning of the type occurring in [3].

Finally we shall prove Theorem 4, which is a special case of a conjectured general Theorem on Convolutions. The conjecture is that if the sequences $\left\{a_{n}\right\},\left\{b_{n}\right\}$ and $\left\{c_{n}\right\}$ defined by (2.0) are related as in (D), then the second differences of $\left\{c_{n}\right\}$ are non-negative if those of $\left\{a_{n}\right\}$ and $\left\{b_{n}\right\}$ are. As in Theorem 1 the sequences may contain zero elements, and in this case the condition of the theorem is understood to apply, in each sequence, up to the first zero term. It will be noticed that in these theorems no mention is made of the structure of the functions represented by the complete power series.

The conjecture would imply a generalisation of both sides of the inequality in [4], since a convex (polygonal) curve necessarily has the properties expressed by these inequalities. It seems also to be true that nothing worthwhile can be said about the third differences of the sequences.

The special case of the conjecture which we prove here is that in which the generating function $f(z)$ of the sequence $\left\{C_{n} / n !\right\}$ is given, namely

$$
f(z)=\prod_{i=1}^{m}\left(1+\alpha_{i} z\right) \quad\left(\alpha_{i}>0, i=1,2, \cdots, m\right),
$$

and this result, of course, generalises Theorem 2(a) of [4]. The method used here depends on the well-known fact that the derivatives of $f(z)$ are of the same form as $f(z)$ itself. Unfortunately, this method will not apply to the parallel case of $g(z)$, say, where

Received by the editors February 2, 1963 and, in revised form, August 15, 1963. 


$$
g(z)=\prod_{i=1}^{m}\left(1-\beta_{i} z\right)^{-1} \quad\left(\beta_{i}>0, i=1,2, \cdots, m\right) .
$$

It would appear that the main difficulty in the way of a proof of the general theorem is its formidable algebraic complexity: the lemmas of Theorem 2 in [1] seem to be relevant.

2. Theorem 1. Let

$$
\left\{a_{n}\right\},\left\{b_{n}\right\},\left\{c_{n}\right\} \quad(n=1,2, \cdots)
$$

be sequences of non-negative numbers and let

$$
A_{0}=1=0 !, \quad A_{n}=\prod_{i=1}^{n} a_{i} \quad(n=1,2, \cdots),
$$

while $B_{n}$ and $C_{n}$ are defined similarly. Suppose

$$
\sum_{r=0}^{n} \frac{A_{r}}{r !} \cdot \frac{B_{n-r}}{(n-r) !}=\frac{C_{n}}{n !} \quad(n=1,2, \cdots) .
$$

Then if

$$
\begin{array}{ll}
\xi \leqq a_{n-1}-a_{n} \leqq \eta & (n=2,3, \cdots), \\
\xi \leqq b_{n-1}-b_{n} \leqq \eta & (n=2,3, \cdots),
\end{array}
$$

where $\xi, \eta$ may be positive or negative, and where ${ }^{1}$

$$
\frac{a_{n-1}}{(n-1)}-\frac{a_{n}}{n} \geqq 0, \quad \frac{b_{n-1}}{(n-1)}-\frac{b_{n}}{n} \geqq 0 \quad(n=2,3, \cdots),
$$

then we shall have

$$
\xi \leqq c_{n-1}-c_{n} \leqq \eta \quad(n=2,3, \cdots) .
$$

The result also holds if the sequences $\left\{a_{n}\right\},\left\{b_{n}\right\},\left\{c_{n}\right\}$ vanish from some point on, provided that in this case we interpret (2.1), (2.2) and (2.4) as holding only for the first zero term and not for later ones.

3. Proof of Theorem 1. Let

$$
\begin{aligned}
& S_{1}(n)=\sum_{0 \leq r \leq s \leq n-1}\left(a_{r+1}-a_{s+1}\right) f(n, r, s), \\
& S_{2}(n)=\sum_{0 \leq r \leq s \leq n-1} \sum_{r+1}\left(b_{r+1}-b_{s+1}\right) g(n, r, s),
\end{aligned}
$$

where

1 The condition (2.3) is automatically satisfied in many cases (see [2]). 


$$
\begin{aligned}
& f(n, r, s)=X_{\bullet}^{(n-1)} X_{r}^{(n-2)}-X_{r}^{(n-1)} X_{\bullet}^{(n-2)} \\
& g(n, r, s)=Y^{(n-1)} Y_{r}^{(n-2)}-Y_{r}^{(n-1)} Y_{\bullet}^{(n-2)}
\end{aligned}
$$

and

$$
X_{r}^{(m)}=\frac{A_{r}}{r !} \frac{B_{m-r}}{(m-r) !} \quad(r=0,1,2, \cdots, m ; m=n-1 \text { or } n-2)
$$

$X_{n-1}^{(n-2)}=0$,

while $Y_{r}^{(m)}$ is defined in exactly the same way, except that the sequences $a_{n}$ and $b_{n}$ are interchanged. Finally let

$$
Q(n)=\sum_{e=1}^{n-2} \sum_{r=1}^{n-1} X_{r}^{(n-1)} X^{(n-2)}
$$

Then it was proved in [1, Lemma 5 et seq. $]$ that the difference $c_{n-1}-c_{n}$ is given by

$$
c_{n-1}-c_{n}=\left(S_{1}(n)+S_{2}(n)\right) / Q(n),
$$

and that the second factor in each term of the sums $S_{1}(n)$ and $S_{2}(n)$ is non-negative if (2.3) is satisfied.

From (2.1) and (2.2) it now follows that

$$
\begin{aligned}
\frac{\xi}{Q(n)}\left\{\sum_{0 \leq r \leq s \leq n-1} \sum_{1}-(r-s)[f(n, r, s)+g(n, r, s)]\right\} \\
\leqq c_{n-1}-c_{n} \\
\leqq \frac{\eta}{Q(n)}\left\{\sum_{0 \leq r \leq s \leq n-1}-(r-s)[f(n, r, s)+g(n, r, s)]\right\} .
\end{aligned}
$$

In the bracketed parts of (3.3) a term like

$$
X_{r}^{(n-1)} X_{r}^{(n-2)}, \quad 0 \leqq r \leqq n-1,0 \leqq s \leqq n-2,
$$

appears with coefficient $(r-s)$ in the sum involving $X$ 's. Since

$$
Y_{t}^{(n-1)} Y_{u}^{(n-2)}=X_{r}^{(n-1)} X_{t}^{(n-2)},
$$

whenever $t=n-1-r, u=n-2-s$, the same term appears in the sum involving $Y^{\prime}$ 's with coefficient $t-u=s-r+1(0 \leqq t \leqq n-1,0 \leqq u \leqq n-2)$. The correspondence thus established between the terms of the first 
and second sums (i.e., the sums involving, respectively, $X$ 's and $Y$ 's) is one-to-one, except for the zero terms $X_{r}^{(n-1)} X_{n-1}^{(n-2)}, Y_{r}^{(n-1)} Y_{n-1}^{(n-2)}$ $(0 \leqq r \leqq n-1)$. Hence

$$
\begin{aligned}
\sum_{0 \leqq r \leqq s \leqq n-1} \sum_{s-s}-(r-s)[f(n, r, s)+g(n, r, s)] & \\
= & \sum_{s=1}^{n-2} \sum_{r=1}^{n-1} X_{r}^{(n-1)} X_{s}^{(n-2)}=Q(n),
\end{aligned}
$$

and so $\xi \leqq c_{n-1}-c_{n} \leqq \eta$ by (3.3).

The truth of the remark about vanishing elements follows when it is noticed that in the definition of $S_{1}(n)$ and $S_{2}(n)$, if $a_{k}=a_{k+1}=0$, then

$$
X_{k}^{(m)}=f(n, k, s)=0 \quad(m=n-1 \text { or } n-2) .
$$

4. Theorem 2. If the reciprocals of the roots of the real polynomial equation

$$
1+a_{1} t+\frac{a_{1} a_{2}}{2 !} t^{2}+\cdots+\frac{a_{1} a_{2} \cdots a_{n}}{n !} t^{n}=0
$$

lie in the region of the Argand diagram defined by

$$
\begin{aligned}
& x^{2}+y^{2}+x \eta \leqq 0, \\
& x^{2}-y^{2}+x \xi \geqq 0,
\end{aligned}
$$

where $\eta \geqq \xi \geqq 0$ and the origin is excepted, then

$$
\xi \leqq a_{i-1}-a_{i} \leqq \eta \quad\left(i=2,3, \cdots, n+1 ; a_{n+1}=0\right) .
$$

NotE. The region concerned is the common part of a circle centred on $(-\eta / 2,0)$ and passing through $(0,0)$ and the left-hand branch of the rectangular hyperbola centred on $(-\xi / 2,0)$, passing through $(0,0)$, and with asymptotes at $45^{\circ}$ to the real axis.

Proof. The conditions (4.2) and (4.3) ensure that the real quadratic or real linear factors of (4.1) are of the form

$$
\begin{array}{ll}
\left(1+b_{1} t+\frac{b_{1} b_{2}}{2 !} t^{2}\right), & \text { where } \xi \leqq b_{1}-b_{2} \leqq \eta, \quad \xi \leqq b_{2} \leqq \eta, \text { or } \\
\left(1+b_{1} t\right), & \text { where } \xi \leqq b_{1} \leqq \eta \text { and } \quad b_{2}=0,
\end{array}
$$

so that the result follows from Theorem 1.

5. Theorem 3. If the roots of the real polynomial equation

$$
1+a_{1} t+\frac{a_{1} a_{2}}{2 !} t^{2}+\cdots+\frac{a_{1} a_{2} \cdots a_{n}}{n !} t^{n}=0
$$


lie in the sector

$$
|\pi-\theta| \leqq \pi / 4
$$

then

(1)

(2) $\frac{a_{r}}{r(n-r+1)} \leqq \frac{a_{r-1}}{(r-1)(n-r+2)}$
$(2 \leqq r \leqq n)$,

$(2 \leqq r \leqq n)$

Proof. If we take $\xi=0, \eta=\infty$ in Theorem 2 , we immediately get (5.3), (1). For the roots, and therefore also the reciprocals of the roots, of (5.1), lie in $|\pi-\theta| \leqq \pi / 4$. On the other hand, by considering the polynomial

$$
1+\frac{n}{a_{n}} t+\frac{n(n-1)}{a_{n} a_{n-1}} t^{2}+\cdots+\frac{n !}{a_{1} a_{2} \cdots a_{n}} t^{n},
$$

which has for its zeros the reciprocals of the roots of (5.1), we see that

$$
\frac{n}{a_{n}} \geqq \frac{2(n-1)}{a_{n-1}} \geqq \frac{3(n-2)}{a_{n-2}} \cdots, \text { etc., }
$$

which is (5.3), (2).

The inequalities (5.3), (1) are better than (5.3), (2) up to about $r=n / 2$. It is worth noting that had the roots of (5.1) been all real and negative then we should have had, by a well-known result ${ }^{2}$

$$
\frac{a_{r}}{(n-r+1)} \leqq \frac{a_{r-1}}{(n-r+2)} \quad(r=2,3, \cdots, n) .
$$

6. Theorem 4. If

$$
f(z)=\prod_{i=1}^{m}\left(1+\alpha_{i} z\right)=\sum_{n=0}^{m} \frac{T(n)}{n !} z^{n}
$$

where $\alpha_{i}>0(i=1,2, \cdots, m)$, then

(6.2) $\frac{T(n+2)}{T(n+1)}+\frac{T(n)}{T(n-1)}-\frac{2 T(n+1)}{T(n)} \geqq 0 \quad(n=1,2, \cdots, m-1)$.

Before giving the proof, which is very simple, we need two lemmas. It will appear, in Lemma 1, that in the case of the function (6.1), the proposition (6.2) has a certain "run-off" property. One is able, in

Theorem 51 , p. 51 of $[5]$. 
effect, to prove only the case $n=1$ of (6.2), and this is accomplished by regarding (6.2) for $n=1$ as part of a special case of the conjecture of $\$ 1$. Thus, in Lemma 2, we shall prove only that the first of the set of second differences of the product sequence $\left\{c_{n}\right\}$ is non-negative, if the same is true of the factor sequences.

LEMMA 1. Let

$$
\begin{aligned}
\frac{d^{j}}{d z^{j}}\{f(z)\} & =\sum_{n=0}^{m-j} \frac{T(j, n)}{n !} z^{n} \\
& =A(j) \prod_{i=1}^{m-j}\left(1+\beta_{i}^{(j)} z\right) \quad(j=0,1, \cdots, m-1),
\end{aligned}
$$

where $A(j)$ is independent of $z$ and $A(0)=1$. Then

(1) $\frac{T(0, n+1)}{T(0, n)}=\frac{T(1, n)}{T(1, n-1)}=\cdots=\frac{T(n, 1)}{T(n, 0)}$, and

(2) $\beta_{i}^{(j)}>0 \quad(i=1,2, \cdots, m-j)$ for $j=1,2, \cdots, m-1$,

if

$$
\alpha_{i}=\beta_{i}^{(0)}>0 \quad(i=1,2, \cdots, m) .
$$

Proof. (1) follows from the definition (6.3). (2) is a well-known result on differentiation.

LEMMA 2. If

$$
\begin{aligned}
& \text { (6.4) } S_{1}=1+a_{1} z+\frac{a_{1} a_{2}}{2 !} z^{2}+\cdots, \quad a_{i} \geqq 0(i=1,2,3 \cdots), \\
& \text { (6.5) } S_{2}=1+b_{1} z+\frac{b_{1} b_{2}}{2 !} z^{2}+\cdots, \quad b_{i} \geqq 0(i=1,2,3 \cdots), \\
& \text { (6.6) } S_{8}=S_{1} \times S_{2}=1+c_{1} z+\frac{c_{1} c_{2}}{2 !} z^{2}+\cdots,
\end{aligned}
$$

then

$$
c_{3}+c_{1}-2 c_{2} \geqq 0,
$$

provided

$$
\begin{aligned}
& a_{3}+a_{1}-2 a_{2} \geqq 0, \\
& b_{3}+b_{1}-2 b_{2} \geqq 0 .
\end{aligned}
$$

Furthermore, the result extends to $m \geqq 3$ factor sequences. 
Proof. If we express $c_{1}, c_{2}, c_{3}$ in terms of $a_{1}, a_{2}, a_{3}$ and $b_{1}, b_{2}, b_{3}$ by means of (6.6) we find that

$$
\begin{aligned}
c_{2} c_{1}^{2}\left\{c_{3}+c_{1}-2 c_{2}\right\} & =a_{2} a_{1}^{2} D(a)+b_{2} b_{1}^{2} D(b) \\
+ & a_{1} b_{1}\left\{2\left[\left(a_{2}-a_{1}\right)-\left(b_{2}-b_{1}\right)\right]^{2}+a_{2} D(a)+b_{2} D(b)\right\},
\end{aligned}
$$

where $D(x)=x_{3}+x_{1}-2 x_{2}$.

Since all the variables are non-negative, the result follows, and it extends by induction to $m \geqq 3$ factor sequences.

Proof of Theorem 4. By Lemma 1 , (1) we have for $1 \leqq n \leqq m-1$,

$$
\begin{aligned}
& \frac{T(n+2)}{T(n+1)}=\frac{T(0, n+2)}{T(0, n+1)}=\frac{T(n-1,3)}{T(n-1,2)}=c_{3} \quad \text { (say), } \\
& \frac{T(n+1)}{T(n)}=\frac{T(0, n+1)}{T(0, n)}=\frac{T(n-1,2)}{T(n-1,1)}=c_{2} \quad \text { (say), } \\
& \frac{T(n)}{T(n-1)}=\frac{T(0, n)}{T(0, n-1)}=\frac{T(n-1,1)}{T(n-1,0)}=c_{1} \quad \text { (say), }
\end{aligned}
$$

where $c_{1}, c_{2}, c_{3}$ satisfy

$$
1+c_{1} z+\frac{c_{1} c_{2}}{2 !} z^{2}+\cdots=\prod_{i=1}^{m-n+1}\left(1+\beta_{i}^{(n-1)} z\right) .
$$

By (2) of Lemma $1, \beta_{i}^{(n-1)}>0(i=1,2, \cdots, m-n+1)$. Hence we can apply Lemma 2 , in the extended form, to obtain the desired result.

Corollary to Theorem 4. As in the case of Theorem 3, it is possible to obtain a meaningful corollary to Theorem 4 by regarding the polynomial from the other end, as a polynomial in $(1 / z)$. The result is complicated to state, however, and we leave it to the reader to deduce for himself.

\section{REFERENCES}

1. J. N. Whiteley, Two theorems on convolutions, J. London Math. Soc. 37 (1962), 459-468.

2. A. Aissen, A. Edrei, I. J. Schoenberg, and A. Whitney, On the generating functions of totally positive sequences, Proc. Nat. Acad. Sci. U.S.A. 37 (1951), 303-307.

3. R. P. Boas, Entire functions, Academic Press, New York, 1954; pp. 23-24.

4. J. N. Whiteley, A generalization of a theorem of Newton, Proc. Amer. Math. Soc. 13 (1962), 144-151.

5. G. H. Hardy, J. E. Littlewood, and G. P6lya, Inequalities, Cambridge Univ. Press, Cambridge, 1934.

Pieterachattzburg, South Africa 